\title{
STATISTICAL AND PHYSICAL ANALYSIS OF THE EXTERNAL FACTORS PERTURBATION ON SOLAR RADIATION EXERGY
}

Juan Carlos Moreno $^{(1)}$, Javier Cañada ${ }^{(2)}$ and José Vicente Boscà ${ }^{(3)}$

(1) and (3) Applied Physics Department

${ }^{(2)}$ Applied Thermodynamics Department

Universidad Politénica de Valencia; Cno.de Vera s/n 46071 Valencia (Spain)

${ }^{(1)}$ E-mail: jcmestev@,fis.upv.es; Fax: (34) $963877329 \quad{ }^{(2)}$ E-mail: jcanada@ter.upv.es; ${ }^{(3)}$ E-mail: jvbosca@,fis.upv.es

Received: 8 November 2002 / Accepted: 9 August 2003 / Published: 31 December 2003

\begin{abstract}
The purpose of this paper is to analyze the external factor perturbations on solar radiation. Firstly, the influence on the photon distribution function in the space of frequencies is analyzed and, later, the modification generated in equations of internal energy, entropy, and radiation is viewed in order to deduce an expression to calculate the spectral exergy of perturbed radiation
\end{abstract}

Keywords: exergy, blackbody radiation, internal energy, entropy.

\section{INTRODUCTION}

Taking into account the negative perspective of our planet's energy resources, the exploitation of solar energy is becoming an urgent need. In order to improve the harnessing of solar energy it is essential to develop an in-depth theoretical knowledge of radiation to understand its behaviour.

The present paper analyses extraterrestrial solar radiation (ESR), usually considered as blackbody radiation, similar to the analysis seen in [1-5]. If the irradiance experimental data, i.e. ASTM [6], is compared with those obtained from model blackbody, slight differences in specific areas of the spectrum is found. This fact surely involves changes in Planck's blackbody equation.

Many authors have analyzed radiation from the quantum, statistical and thermodynamic point of view. For example, Smirnov [7] presented an original Heisenberg-picture approach to a brownian motion of 
wave packets in a dissipative environment, also he studied the interaction of constant uniform magnetic field with the classical blackbody radiation heat bath and analyzed the special features of wave packet spreading and reduction stemming from the non-white nature of the noise from the classical blackbody radiation heat bath. Mandal [8] presented a graphical method to obtain the photon number distribution

for squeezed states of real parameters. The experimental values of photon number distribution agrees well with the method reported. Miyashita et al. [9] demostrated that a sub-Poissonian distribution of photon numbers (photon number squeezing) within a microcavity is theoretically shown to be possible using a realistic model of nonlinear photon dissipation. Cheng [10] has analyzed the quantum effects of thermal radiation in a Kerr nonlinear blackbody. Barbosa et al. [11] have investigated the generational and statistical properties of the generalized superposition of two squeezed states, and have studied the nonclassical properties of a generalized superposition state which interpolates between two arbitrary squeezed states as a function of the interpolating parameters.

Such analysis is based on the statistical thermodynamic treatment of blackbody radiation, being the main objective to find an expression capable of providing the useful work that we can extract from radiation, resorting to the concept of exergy, which comprises magnitudes of internal energy, entropy, and radiation pressure. The following sections analyze the perturbation produced by external phenomena on the thermodynamic magnitude equations. From the Anaxagoras' principle quotation, where everything is mixed with everything, it is obvious that several external factors to radiation such as solar wind, magnetic fields, Van Allen belts, and gravitational fields, will influence the photon displacement from the Sun to the Earth.

Firstly, the analysis of the perturbation of external factors in the photon distribution function in the space of frequencies. Secondly, it is viewed in the modification generated in the equations of internal energy and radiation spectrum entropy. Thirdly, the appropriate modification on the radiation pressure is also studied. And lastly, the equation of the spectrum exergy of the blackbody radiation, with or without perturbation, is deduced.

\section{PHOTON DISTRIBUTION FUNCTION}

This section analyzes the effect of external factors on the solar radiation photon distribution function. Until now, several authors have idealized the behaviour of solar radiation, emphasizing for example Jeter's [1] suppositions:

- considering ESR with a blackbody distribution of a maximum of $0.5 \mu \mathrm{m}$ representative of a temperature source near $5800 \mathrm{~K}$.

- supposing that the Sun is a uniform luminous source with a $0.01 \mathrm{rd}$. diameter, and consequently, the speed of the photons falling on the Earth's orbit, it can be considered that their direction is distributed within a $0.01 \mathrm{rd}$ angle cone. With this supposition, the darkening effect of the solar disk is disregarded.

Photons comply with the Bose-Einstein statistics. They present the fundamental characteristics of indistinguibility and non-interaction among them, giving an indefinite total number in the flow. Their physical distribution is represented if energy levels, which, at the same time, can be subdivided into cells called quantum states,(see Rumer et al.[7] and Matveev [8]). 
Let's suppose that we have $Z$ quantum states separated by Z-1 partitions of the energetic level, where we distribute $N$ photons. The number of $Z-1+N$ elements, $N$ representing particles and $Z-1$ partitions, is hence obtained. When all the possible changes in these elements have been carried out we obtain all the possible distributions of $N$ particles among the $Z$ cells. This number of distributions should be decreased $N$ ! times since the changes of supposedly identical particles do not affect the production of new distributions. A decrease of (Z-1)! times must be applied, since the changes in cell borders do not lead to different distributions. Therefore, the number of distributions of the photons among the $\mathrm{Z}$ quantum states equals,

$$
\frac{(Z+N-1) !}{N !(Z-1) !}
$$

This distribution is produced in each of the energetic bands of the photon system. We will apply to all bands the product of all distributions from each energetic level, which corresponds to the statistical weight of the total macroscopic state of the system.

$$
\Gamma=\prod_{i} \frac{\left(Z_{i}+N_{i}-1\right) !}{N_{i} !\left(Z_{i}-1\right) !}
$$

A state of equilibrium requires that the number of $\Gamma$ states be should be maximum, that is that the most probable distribution is searched. For reasons of simplicity, the maximum $\ln \Gamma$ is determined by previously adding two supplementary conditions:

a) the total number of particles is given by,

b) the total energy of the system is

$$
\sum_{i} N_{i}=N
$$

$$
\sum_{i} N_{i} \varepsilon_{i}=E
$$

being $\varepsilon_{i}$ the energy at each level. Using Lagrange's multipliers method, it is obtained as an extreme condition of the equation,

$$
\frac{\partial \ln \Gamma}{\partial N_{i}}-\beta \varepsilon_{i}-\alpha=0
$$

being " $\alpha$ " and " $\beta$ " Lagrange's multipliers. Since the values in the number of cells and photons is high, we can apply in Eq.(2) Stirling's formula: $\ln N !=N \ln N-N$ and also, in the case of the photons, the condition of the constancy in the number of photons Eq.(2) is cancelled out and consequently the value of " $\alpha$ " due to the function partition used in statistical physics is $\Omega=\sum_{R} e^{-\beta E_{R}}$; where $\mathrm{R}$ is whole number of states, and Reif [14] showed that the parameter $\alpha$ is related to the function partition by the relationship, $\alpha=\frac{\partial \ln \Omega}{\partial N}$. For the photons, there are no limitations in the total number of particles, this involves that the function partition is independent of $\mathrm{N}$; hence $\alpha=0$. With all this, the equation (5) is 
transformed into,

$$
\ln \left(\frac{Z_{i}}{N_{i}}+1\right)-\beta \varepsilon_{i}=0
$$

and finding the quotient $N_{i} / Z_{i}$ Planck's distribution can be deduced

$$
\bar{n}\left(\varepsilon_{i}\right)=\frac{N_{i}}{Z_{i}}=\frac{1}{e^{\beta \varepsilon_{i}}-1}
$$

The external factors mentioned in the introduction must slightly disturb the state of equilibrium of the radiation, since the comparison of experimental data from the ESR with those from the blackbody present many similarities. One of the possible changes will be due to the action of the gravitational field which will induce a curve in the trajectory of photons. The application of the general theory of relativity could explain the perturbations on the photon flow. However, this section intends to find a justification from the quantum mechanics approach. The effect of an external field on the photon flow will not modify in any way Planck's distribution Eq.(7). We must necessarily consider a influence on the source of the distribution equation that is in the statistical distribution Eq.(1). The hypothetical influence acts at the quantum state level of the photons, in a way that we can figure out two alterations in reference to the equilibrium situation:

a) reduction of the number of cells in each energetic level.

b) splitting of the number of cells in each energetic level.

The first case implies a restriction in the mobility of the photons set, which in terms of intuition, decreases possibilities for action to the system. In terms of energy, we can state that there will exist less capacity for work.

As it has been commented above, the system has $\mathrm{Z}$ quantum states (also denominated cells). The disturbing external field acts upon the quantic configuration, changing the energetics levels, cells and number of particles in each cell. Supposing now a reduction of $\mathrm{M}$ cells in each energetic level, the substraction of a random $M$ number of cells transforms equation (2) into,

$$
\Gamma r=\frac{(Z+N-M) !}{N !(Z-M) !}
$$

being $\Gamma r$ the possible number from the photon distribution under these circumstances of reduction. If we apply Stirling's formula next; calculating the neperian logarithmn and derivating $N$, we obtain, 


$$
\ln \frac{(Z+N-M) !}{N !}
$$

which added to the equation (6)

$$
\ln \left(\frac{Z_{i}}{N_{i}}+\frac{\left(N_{i}-M\right)}{N_{i}}\right)-\beta \varepsilon_{i}=0
$$

Rewriting the quotient $N_{i} / Z_{i}$ we find that the distribution in the case of a reduction in the quantum states, (what similarities or differences between the $\bar{n} r(\varepsilon)$ and $\bar{n}(\varepsilon)$ have not been explained clearly)

$$
\bar{n} r\left(\varepsilon_{i}\right)=\frac{N_{i}}{Z_{i}}=\frac{1}{e^{\beta \varepsilon_{i}}-\left(\frac{N_{i}-M}{N_{i}}\right)}
$$

Being the $\left(N_{i}-M\right) / N_{i}$ quotient slightly inferior than the unit. The differences between the functions of distribution in equilibrium and alteration is obtained from the subtraction of rate $\mathrm{M} / \mathrm{N}_{\mathrm{i}}$ which represents a decrease of cells according to the number of particles, as we suppose that the two hypothesis in this paper, namely: a) the same reduction or splitting of cells in each energetic level, and b) the same decrement of number photons (or exchange) in each energetic level.

If we imagined a splitting of quantum states due to an external factor we would have the following statistical distribution,

$$
\frac{(Z+N+M) !}{N !(Z+M) !}
$$

If we repeat the process in the case of reduction we obtain the distribution,

$$
\bar{n} d\left(\varepsilon_{i}\right)=\frac{N_{i}}{Z_{i}}=\frac{1}{e^{\beta \varepsilon_{i}}-\left(\frac{N_{i}+M}{N_{i}}\right)}
$$

being the quotient $\left(N_{i}+M\right) / N_{i}$ slightly superior to the unit.

Since $\mathrm{M}$ is much smaller than $\mathrm{N}_{\mathrm{i}}$ the separation from the equilibrium situation is small. The next section studies the thermodynamic consequences of perturbation in the distribution function. 


\section{INTERNAL ENERGY FUNCTIONS AND RADIATION SPECTRUM ENTROPY}

This section presents the alteration to equations of the mentioned reduction and splitting of photon quantum states.

Badescu [15] suggested an expression for the internal energy of blackbodies at $5777 \mathrm{~K}$ according to frequency $\left(\mathrm{J} \cdot \mathrm{m}^{-3} \cdot \mathrm{Hz}^{-1}\right)$.

$u_{v}=\frac{h}{c^{3}} \frac{v^{3}}{e^{\beta v}-1} \pi(1-\cos 2 \delta)$

where $h$ is Planck's constant; $c$ is the speed of light; $\beta=h / k T(T=5777 \mathrm{~K})$; and $\delta$ is the observation semiangle of the solar disk from the Earth.

We suggest that if the distribution functions (11) and (13) are incorporated to equation (14) the internal energy is obtained for the reduction and splitting of quantum levels.

$$
u_{v}=\frac{h}{c^{3}} \frac{v^{3}}{e^{\beta v}-x} \pi(1-\cos 2 \delta)
$$

being $x=\frac{N_{i}-M}{N_{i}}$ the term mentioned above in equation (11). It is $\mathrm{x}<1$ in the case of reduction. On the contrary, in equation (13) the term $\mathrm{x}$ has the expression, $x=\frac{N_{i}+M}{N_{i}}$. It is $\mathrm{x}>1$ in the case of splitting.

Supossing values of term $\mathrm{x}=\{0.5,1.5\}$ for reduction and splitting respectively, the alterations of internal energy values can be seen in figure 1. For strong splitting of quantic configuration, the curve of internal energy presents a maximum in low frequency $\left(0.1 \cdot 10^{15} \mathrm{~Hz}\right)$ and, on the other hand, the rank of frequency is reduced since they are negative values at the proximity of origin, that has no physical meaning. The change in quantic splitting causes an increase of energy but in a lower spectrum rank. As it can be seen, the effect is much more sensitive in the low frequency area, where the photons are less energetic, and logically are more perturbable by an external field.

Such values, $x=\{0.5,1,5\}$, suposses there is a $50 \%$ splitting or reduction in the number of cells of the quantum configuration. It is just a supossed value to graphically illustrate more clearly the effect studied. 
The splitting consists of an increase in the number of cells which provides a higher performance capacity, that is, more energy. If we compare Iqbal's experimental data (1983) [6] with those of the blackbody (figure 2) we can distinguish two areas:

- From $1.5 \cdot 10^{15} \mathrm{~Hz}$ to $0.75 \cdot 10^{15} \mathrm{~Hz}$ there is a slight reduction of quantum states.

- From $0.75 \cdot 10^{15} \mathrm{~Hz}$ to low frequencies there is splitting.

A possible justification of the quantum perturbations could be as follows: we start with a control volume with internal electromagnetic radiation which is spreading from one point to another. When entering a force field inside the volume, the resonance effect can take place, coinciding with "valleys and

peaks" of perturbing and electromagnetic waves producing, as a last resort, a constructive interference which produces an increase of propagation directions of the waves within the control volume. Such interference actually produces an extension of possible existing states of photons; in other words, it increases the quantum configuration with the contribution of the perturbing field itself.

In the case of high energy photons a reaction of quantum states takes place, being the destructive interference of electromagnetic waves a plausible argument, which eliminates some photons. This elimination means a suppression of a number of transit directions within the control volume.

Therefore, a restriction in the capacity of the photon flow performance is generated, or in other words, a loss of quantum states.

The perturbation of the function entropy of the radiation is studied. Landau [16] suggested an equation to calculate the entropy of the radiation per volume unit and frequency:

$$
s_{v}=k \cdot\left[\left(n_{v}+1\right) \ln \left(n_{v}+1\right)-n_{v} \ln n_{v}\right] \frac{2}{c^{3}} v^{3} \cdot \Omega \cdot \cos \delta
$$

where, A $\delta @$ is the observation semiangle of the solar disk from the Earth, whose value in comprised between 0 and 0.00465 radians; $\Omega$ is the solid angle subtended on the Earth which receives all the solar disk; $n_{v}$ is Planck's distribution function Eq.(7) which is expressed according to frequencies; $k$ is Boltzmannn constant and $c$ the speed of light in a vacuum.

Alternatively, the distribution of perturbed functions (11) and (12) are substituted in the equation (16) and the original functions are calculated, obtaining the spectrum entropy per unit of the volume of the radiation with reduced quantum and splitted states respectively. In figure 3 such equations are represented, including the equation of blackbody radiation without perturbing.

It is seen that a higher entropy in the case of quantum splitting, since photons achieve a higher number of range probabilities that exist in the quantum flow configuration. Quite the contrary, lower quantum states, as in the case of reduction of the lower range probabilities exist which corresponds to a lower entropy. 
This part of the section has demostrated that the internal energy as well as low frequency blackbody radiation entropy presents higher values when there exists quantun level splitting. The opposite takes place in quantum levels reduction.

The function distribution of photon is decreasing exponentially. The majority of particles are placed around low frequencies, and augment the probability of interaction. When the frequency is increased all the functions distribution (black body, black body splitting, and black body reduction) tend towards the same values. Hence the probability of interaction is similar, and consistently the same efects.

\section{RADIATION PRESSURE}

The calculation of exergy requires previous knowledge of the internal energy, entropy and radiation pressure variables. This section studies the influence of the perturbation of the number of quantum states on the radiation pressure function. As in previous sections, three different situations are analyzed: blackbody radiation without perturbation, blackbody radiation with splitting and reduction of quantum states number.

The average pressure of the radiation on the enclosure walls is given by Reif, [14]:

$$
\bar{p}=\sum_{s} \bar{n}_{s}\left(-\frac{\partial \varepsilon_{s}}{\partial V}\right)
$$

in which, $n$ and $\varepsilon$ are the distribution functions and photon energy, and the subindex As@ refers to the energy levels of photons.

In order to obtain $-\frac{\partial \varepsilon_{s}}{\partial V}$ let us consider, for the sake of simplicity, that the enclosure is a cube with an $\mathrm{L}$ edge. The condition of presenting the same value of input and output from the control volume is imposed over the photon wave function, which removes the problem of reflection on the enclosure walls and creates the quantification of the number of waves and, at the same time, the photon energy, (see Reif [14] )

$$
\varepsilon_{s}=h v=\frac{h}{2 c}\left(\kappa_{x}^{2}+\kappa_{y}^{2}+\kappa_{z}^{2}\right)^{1 / 2}
$$

being $\kappa_{i}$ the components of the number of waves restricted to whole, positive, negative or Am@ null values; called quantum numbers; according to the equation, (see Reif [14] )

$\varepsilon_{s}=\frac{h c}{L}\left(m_{x}^{2}+m_{y}^{2}+m_{z}^{2}\right)^{1 / 2}=C \cdot L^{-1}=C \cdot V^{-1 / 3}$ 
where $C$ is a constant which comprises quantum numbers, Planck's constant and the speed of light $c$.

Now the partial derivative of energy in reference to volume can be calculated.

$$
\frac{\partial \varepsilon_{s}}{\partial V}=-\frac{1}{3} C V^{-\frac{4}{3}}=-\frac{1}{3} \frac{\varepsilon_{s}}{V}
$$

Substituting in (17) we obtain,

$$
\bar{p}=\frac{1}{3 V} \sum_{s} \bar{n}_{s} \varepsilon_{s}
$$

The present paper is interested in working with spectrum magnitudes. Hence, it is written with an equation of spectrum pressure radiation as one third of the internal energy Eq.(15), but with the quotient $\zeta$ calculated at a temperature of $298.15 \mathrm{~K}$, since the pressure of radiation is in dead state must be calculated. We study, as in the previous magnitudes, the effects of quantum levels of reduction and splitting. These pressures are the order $10^{-27}$, and for that reason they do not affect the calculation of the exergy.

The pressure of radiation with quantum state splitting is higher especially at lower frequencies. Probably, the delocalization of photons when increasing their quantum configuration range produces a higher number of impacts in the walls of the control volume. On the contrary, a lower pressure in the case of reduction, may be caused by a decrease in the number of impacts with the enclosure walls because they have a lower quantum state range that has a lower capacity of circulation.

\section{RADIATION SPECTRUM EXERGY}

The spectrum exergy per volume unit $\left(\mathrm{J} \cdot \mathrm{m}^{-3} \cdot \mathrm{Hz}^{-1}\right)$ has the equation (from reference [15]):

$$
e x_{v}=u_{v}-T_{o} \cdot s_{v}+p_{o v}
$$

where the temperature and pressure refer to the dead state: $298.15 \mathrm{~K}$ and $1 \mathrm{~atm}$ being this pressure of a photon average value for each frequency.

If we substitute the appropriate internal energy, entropy and radiation pressure equations for the three cases of quantum configuration mentioned above, we obtain the spectrum exergy of figure 4 . It can be deduced that the radiation exergy with splitting is higher at low frequencies, while it is the opposite for higher frequencies. The point of exergy value inversion between the configurations of 
splitting and reduction is $0.5 \cdot 10^{15} \mathrm{~Hz}$, due to the form of the entropy equations (see figure 3 ). In figure 5 the percentage deviation of blackbody radiation is analyzed in regards to the equilibrium and also is represented by the deviation of the solar radiation experimental values. These values are subtracted previously from the two sources of the variation in extraterrestrial radiation: periodic variations of intrinsic solar radiation and the variation of the earth-sun distance; both sum up approximately $5 \%$, see Duffie and Beckman [17]. If is supposed that the rest of the deviation is produced in changes of quantic distribution, creating two wavelengths of maximal quantic perturbation: 1.6 and $2.7 \mu \mathrm{m}$.

\section{CONCLUSIONS}

A model to explain the way in which external factors affect the distribution of photons has been suggested. This model is based on quantum cell splitting and on the reduction of energy levels.

The consequences on internal energy, entropy and radiation pressure have been studied, the fact of finding two large areas in the experimental spectrum extraterrestrial irradiance confirms this idea: There is an area of quantum states splitting at low frequencies, and an area of quantum state reduction at higher frequencies.

Internal energy, entropy and spectrum radiation pressure present higher values when there is quantum level splitting. It may be due to the fact that the increase in the quantum configuration of the control volume provides a higher capacity for photon activity.

The spectrum exergy function presents two delimited areas at the $600 \mathrm{~nm}$-axis of figure 4 . In each area there is a predomination of a specific exergy function: splitting at lower frequencies, and reductions at higher frequencies, although it is not so pronounced. The reason for the shape of the spectrum entropy (see figure 3 ) is due to eq. 22 as the $2^{\text {nd }}$ term of the right member is the largest at greater frequencies, thus producing higher values of exergy.

At $600 \mathrm{~nm}$ the spectral curve remains divided in two regions: zone with quantic splitting and zone of quantic reduction referring to the values of the equilibrium curve of blackbody radiation. The point of $600 \mathrm{~nm}$ is the intersection of three curves b.b.,b.b.s.; b.b.r. and this represents a point of equilibrium. The alteration of the external field on the radiation looks like a turn around of the whole curve in the hourly direction in reference to the afixed point. 


\section{NOMENCLATURE}

$c \quad$ speed of light in a vacuum $: 3 \cdot 10^{8} \mathrm{~m} \cdot \mathrm{s}^{-1}$

$E$ total energy system: $\mathrm{J}$

ex exergy radiation; $\mathrm{J} \cdot \mathrm{m}^{-3} \cdot \mathrm{Hz}^{-1}$

$h$ Planck's constant: $6.62617 \cdot 10^{-34} \mathrm{~J} \cdot \mathrm{Hz}^{-1}$

$k$ Boltzmann's constant: $1.38066 \cdot 10^{-23} \mathrm{~J} \cdot \mathrm{K}^{-1}$

$\kappa$ number of waves

$m$ quantum numbers

$h \quad$ Planck's distribution

$N$ number of photons

$p \quad$ radiation pressure: $\mathrm{N} \cdot \mathrm{m}^{-2}$

$s$ entropy of radiation: $\mathrm{J} \cdot \mathrm{m}^{-3} \cdot \mathrm{Hz}^{-1} \mathrm{AK}^{-1}$

$T$ temperature of radiation; $\mathrm{K}$

$u$ internal energy radiation: $\mathrm{J} \cdot \mathrm{m}^{-3} \cdot \mathrm{Hz}^{-1}$

$V$ control volume of radiation: $\mathrm{m}^{3}$

$Z$ quantum states

\section{GREEKS:}

$\alpha \quad$ Lagrange's multipliers

$\beta \quad$ constant $=(k T)^{-1}$

$\delta \quad$ the observation semiangle of solar disk from the Earth: $0.00465 \mathrm{rd}$.

$\Gamma$ possible quantum states system

$\varepsilon \quad$ state energy

$\zeta \quad$ constant $=h \cdot k^{-1} T^{\mathrm{B} 1}$

$\mathrm{v}$ frequency

$\Omega$ solid angle subtended on the Earth which receives all the solar disk

\section{SUBINDEX:}

$o$ dead state

$s$ energy state 


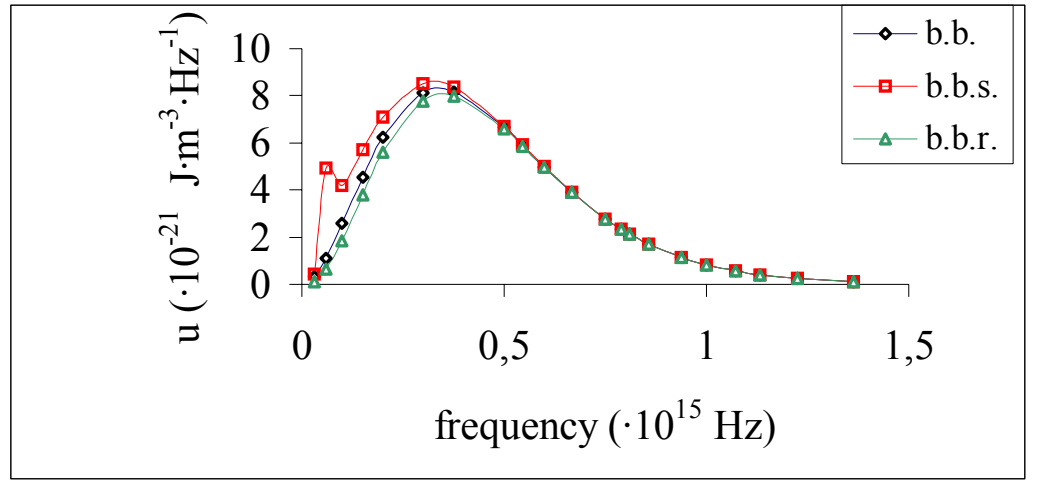

Figure1.-Radiation spectrum internal energy: 1) blackbody (b.b.); 2) blackbody with splitting (b.b.s.) and 3) blackbody with reduction (b.b.r.).

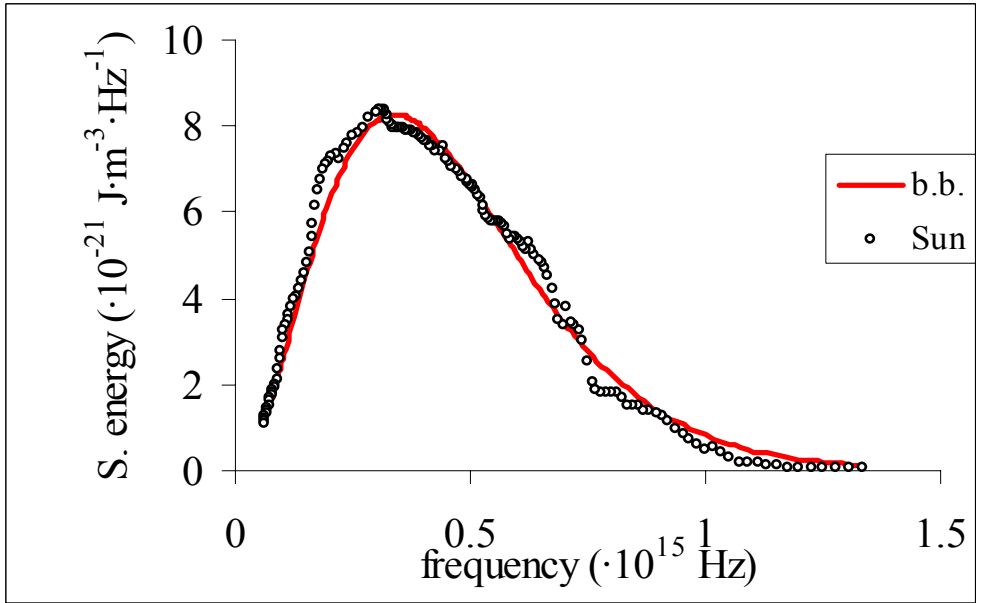

Figure 2.- Sun and blackbody spectrum energy versus frequency. 


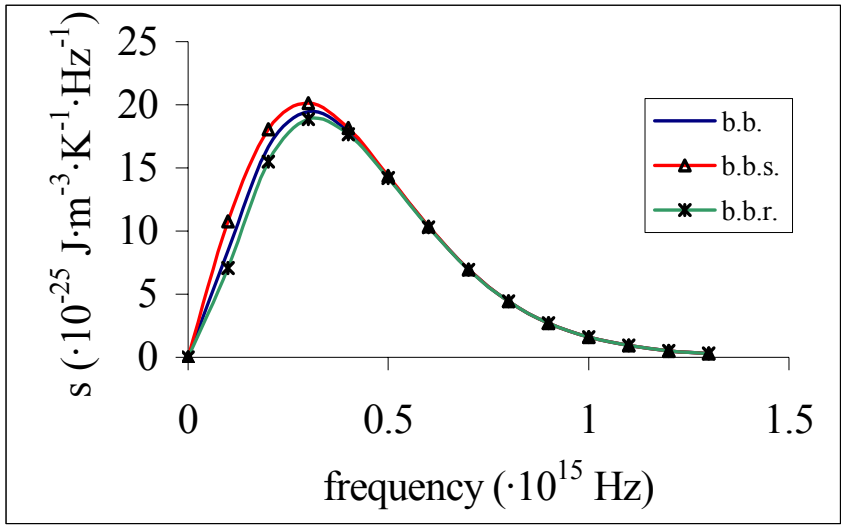

Figure 3.- Radiation spectrum entropy: 1) blackbody (b.b.); 2) blackbody with splitting (b.b.s.) and 3) blackbody with reduction (b.b.r.).

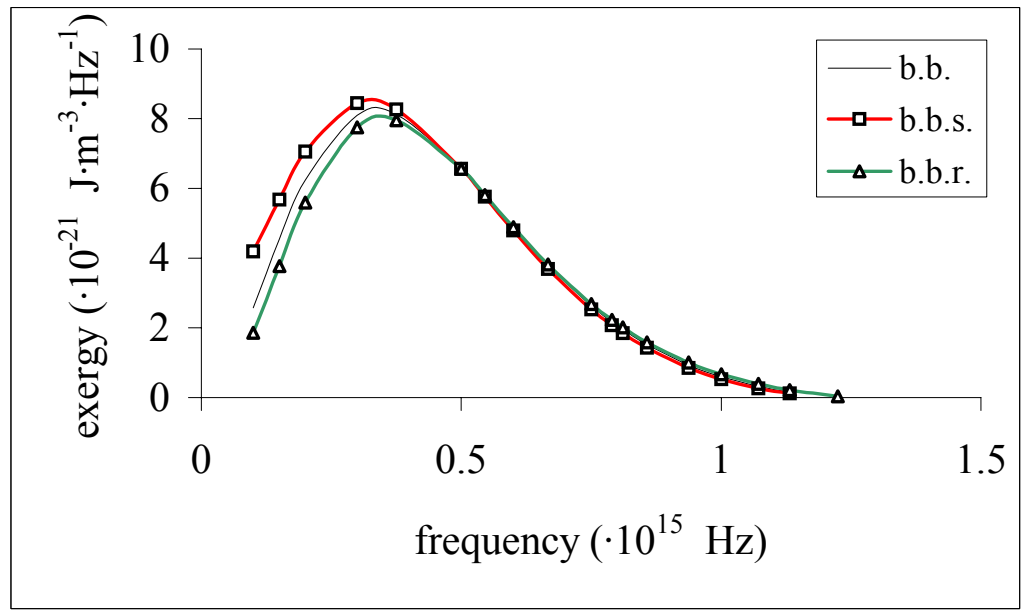

Figure 4.- Radiation spectrum exergy: 1) blackbody (b.b.); 2) blackbody with splitting (b.b.s.) and 3) blackbody with reduction (b.b.r.). 


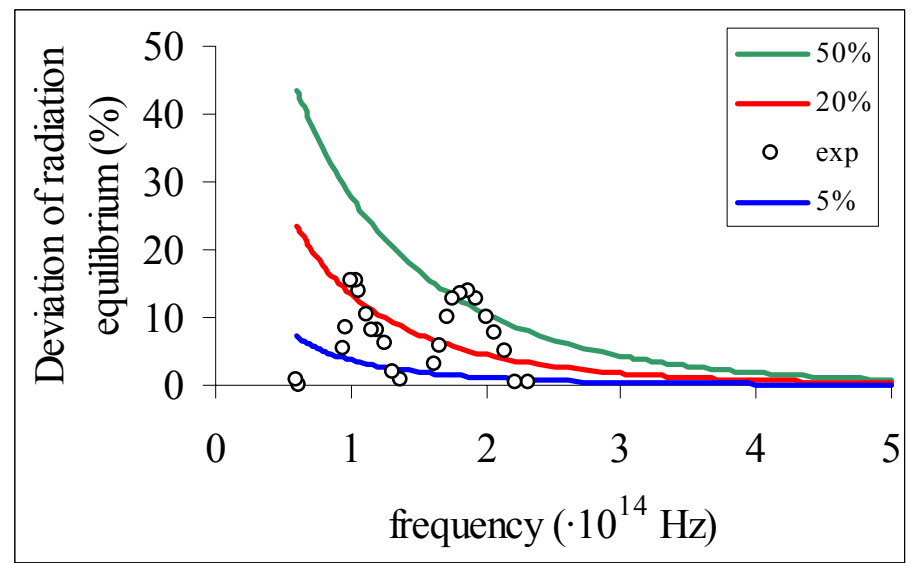

Figure 5.- Deviation of radiation equilibrium (\%) versus frequency. The labels correspond to percentage change of photons configuration as regards equilibrium, and the experimental values. 


\section{REFERENCES}

1. Jeter, S. Maximum Conversion Efficiency for the Utilization of Direct Solar Radiation. Solar Energy 26, 231-236, (1981).

2. Parrott, J.E. Theoretical upper limit to the Conversion Efficiency of Solar Energy. Solar Energy, 21, 227-233,(1978).

3. Spanner, D.C., Introduction to Thermodynamics. Academic Press, New York (1964).

4. Petela, R. Exergy of Heat Radiation. J. Heat Transfer, 86, 187-192,(1964).

5. Press, W.H. Theoretical Maximum for energy from Direct and Diffuse sunlight. Nature, 264,735, (1976).

6. ASTM.E490-73a 'Standard Solar Constant and Air Mass Zero Solar Spectral Irradiance Tables', (1992).

7. Smirnov, A.Y. 'Radiation-induced reduction of a wave packet in a magnetic field' Physica A, 253 (1-4) : 161-177 May 1 (1998).

8. Mandal, S. 'Photon-number distribution of squeezed states: A graphical treatment'. Physical Review A, 58(1), 752-754 Jul (1998).

9. Miyashita, S. ; Ezaki, H. ; Hanamura, E. 'Stochastic model of nonclassical light emission from a microcavity'. Physical Review A, 57 (3): 2046-2055 Mar (1998).

10. Cheng, Z. 'Quantum effects of thermal radiation in a Kerr non-linear blackbody'. Journal

of the Potical Society of America B - Optical Physics, 19(7): 1692-1705 Jul (2002).

11. Barbosa, Y.A.; Marqués, G.C. ; Baseia, B. 'Generalized superposition of two squeezed states: generation’ Physica A, 280 (3-4): 346-361 (2000)

12. Matveev, A.N. Molecular Physics. Ed. Mir, Moscú (1985).

13. Rumer, Y.B., Ryvkin, M.Sh., Thermodynamics Statistical Physics and Kinetics. Ed. Mir, Moscú, (1980).

14. Reif, F. Fundamentos de Física Estadística y Térmica. Ed.del Castillo, Madrid, (1974).

15. Badescu, V., L'Exergie de la Radiation Solaire Directe et Diffuse sur la surface de la Terre,Entropie 145, 67-72,(1988).

16. Landau, I. Física Estadística. Ed. Mir, Moscú, (1967)

17. Duffíe, J.A., Beckman, Solar Engineering of Thermal Process. Wiley-Interscience Publication,(1980)

(C) 2003 by MDPI (http://www.mdpi.org). Reproduction for noncommercial purposes permitted 
